\title{
Effect of Composite Material Parameters on Vibrational Behaviour of Pipes Conveying Fluid
}

\author{
E. Rabeih ${ }^{\dagger}$, M. El-Maddah, R. Gadelrab and A. Atwa \\ Helwan University, Faculty of Engineering-Mataria, Masakin Elhelmia, PO 11718, Cairo, Egypt
}

(Received 28 April 2004; accepted 16 March 2005)

\begin{abstract}
High flow velocity causes instability in pipes. The critical flow velocity, which causes the onset of the pipe's instability, depends on the mechanical and physical parameters of its material. For pipes made of fibre composite materials, these properties depend on composite material parameters such as the fibre orientation angle and fibre volume fraction. The effect of these parameters on the natural frequencies and critical flow velocities of composite material pipes has been investigated for different pipe configurations. A finite element model derived based on the Timoshenko beam theory for a generally orthotropic material pipe has been used in this paper. The results showed that the critical flow velocity is greatly affected by the composite material parameters.
\end{abstract}

${ }^{\dagger}$ Member of the International Institute of Acoustics and Vibration (IIAV)

\section{Nomenclatures}

A $\quad-$ cross section of the beam

$A_{f} \quad-$ cross section area of the fluid

$A_{p} \quad-$ cross section area of the pipe

$C_{t} \quad$ - torsional rigidity

$C_{m t} \quad-$ mutual torsional rigidity

$G_{i j} \quad$ - modulus of rigidity $(i, j=x, y)$

$E_{i j} \quad$ - Young's modulus $(i, j=x, y, z)$

$E_{\text {fibre }}-$ Young's modulus of fibre

$E_{\text {matrix }}$ - Young's modulus of matrix

$I_{f} \quad-$ moment of inertia of fluid

$I_{x} \quad-$ inertia moment of the cross section

$J_{x z} \quad-$ polar moment of inertia

$K E \quad-$ kinetic energy

$L \quad$ - length of element

$L_{p} \quad-$ length of the pipe

$m_{f} \quad$ - fluid mass

$M \quad$ - bending moment

$Q \quad-$ shear force

$S_{i j} \quad-$ compliance matrix factor $(i, j=1 \div 6)$

$T_{m} \quad-$ twisting moment

$U \quad$ - potential energy

$v \quad-$ fluid flow velocity in $\mathrm{m} / \mathrm{s}$

$u \quad-$ dimensionless flow velocity, $v L_{p} \sqrt{m_{f} / E_{z z} I_{x}}$

$y \quad$ - lateral displacement

$\theta \quad-$ fibre orientation angle

$\psi \quad-$ bending slope of the cross section

$\lambda-$ dimensionless natural frequencies, $\omega L_{p}^{2} \sqrt{\rho A / E_{z z} I_{x}}$

$\phi \quad-$ twisting angle of the cross section

$\mu_{i j} \quad$ - Poisson's ratio $(i, j=x, y)$

$\mu_{\text {fibre }}-$ Poisson's ratio of the fibre

$\mu_{\text {matrix }}-$ Poisson's ratio of the matrix

$\rho-$ density of the beam material

$\rho_{p} \quad-$ density of the pipe material

$\rho_{f} \quad-$ fluid density

$\omega \quad$ - radial natural frequency

\section{INTRODUCTION}

It is well known that a pipe conveying fluid becomes unstable when the flow velocity exceeds a critical value. This critical flow velocity depends on the physical and mechanical properties of the pipe material. However, the properties of the pipe material, especially composite material pipes, depend on the characteristics of the material, whether it is isotropic or not. ${ }^{1}$

Traditional materials are, in general, isotropic materials which have direction-independent properties. Therefore, the equation of motion of the transverse vibration is a fourthorder differential equation. In a finite element model of transverse vibration of traditional materials, a two-node element with two degrees of freedom at each node is usually enough. However, fibre composite materials are generally orthotropic materials which have direction-dependent properties due to the effect of the fibre orientation angle. ${ }^{2}$ In these materials, the flexural and torsional deflections are coupled; consequently, the bending and torsional vibration modes are coupled. Therefore, the additional degree of freedom at each node represents the torsional displacement that should be considered in the finite element model for the transverse vibration of composite material pipes.

The dynamic behaviour of generally orthotropic material beams was extensively studied to illustrate the effect of the fibre orientation angle on the natural frequency and mode shape of such beams. ${ }^{3-6}$ The effect of flow velocity on the natural frequency and critical flow velocity has also been extensively studied for an isotropic material ${ }^{7-10}$ and especially for an orthotropic material, which has no coupling between the torsional and flexural deflection.

Composite material pipes are widely used in conveying fluid because of the well-known advantageous of these materials over traditional materials. ${ }^{1}$ These pipes may be manufactured with different configurations of fibre orientations and fibre volume fraction in order to satisfy certain strength requirements. However, these configurations affect their natural frequencies and the critical flow velocity, which may causes the onset of the pipe's instability at the applied flow 